\title{
Bacterial immune evasion via an IL-10 mediated host response - a novel pathophysiologic mechanism for chronic rhinosinusitis*
}

\author{
Joseph S. Schwartz",\#, Sawsan Al-Mot',", Leandra Mfuna Endam², \\ Saud Alromaih ${ }^{1,3}$, Joaquin Madrenas ${ }^{4}$, Martin Desrosiers ${ }^{2,5}$ \\ ' Department of Otolaryngology - Head \& Neck Surgery, McGill University, Montreal, Canada \\ ${ }^{2}$ Centre de Recherche du Centre Hospitalier de I'Université de Montréal (CRCHUM), Montreal, Canada \\ ${ }^{3}$ Department of Otolaryngology -Head and Neck Surgery, Faculty of Medicine, King Saud University, Riyadh, Saudi Arabia \\ ${ }^{4}$ Department of Microbiology \& Immunology, McGill University, Montreal, Canada \\ ${ }^{5}$ Department of Otolaryngology - Head \& Neck Surgery, Université de Montréal, Montreal, Canada
}

Rhinology 55: 227-233, 2017

https://doi.org/10.4193/Rhino16.199

* Received for publication:

June 10, 2016

Accepted: January 11, 2017

\# These two authors contributed

equally to this work

\begin{abstract}
Background: Staphylococcus aureus is a frequently implicated pathogen in chronic rhinosinusitis (CRS). S. aureus may promote commensalism by downregulating pro-inflammatory T cell host responses via an IL-10 mediated pathway. This finding, coupled with the observation that $S$. aureus and $C D 8^{+} T$ cell numbers are inversely correlated in CRS mucosa, suggests that $S$. aureus may evade immune destruction via IL-10 induction. To support this hypothesis, we evaluated i) whether IL-10 levels differ in CRS compared to controls (CTL) using microarray and immunohistochemistry and ii) whether IL-10 levels correlate with S. aureus and CD8 T cell levels.
\end{abstract}

Methodology: Sinus epithelial brush samples from 12 patients undergoing ESS for CRS and 10 CTLs underwent microarray analysis of IL-10 gene expression. Microarray results were verified on simultaneously obtained surgical biopsy samples by immunohistochemistry staining for IL-10. Potential mechanisms were assessed by immunohistochemistry for $\mathrm{CD}^{+} \mathrm{T}$ cells and S. aureus.

Results: IL-10 gene expression was significantly higher in CRS vs CTL subjects at the time of surgery. Immunohistochemistry confirmed increased levels of intraepithelial IL-10. A strong inverse correlation was observed between intraepithelial IL-10 and CD8 ${ }^{+} T$ cell levels as was intraepithelial IL-10 and S. aureus.

Conclusion: Elevated IL-10 levels in sinus mucosa may be a potential pathophysiologic feature of CRS in association with a significant downregulation of host $\mathrm{CD} 8^{+} \mathrm{T}$ cell levels. While $\mathrm{S}$. aureus is believed to play a role in IL-10 induction, a comparatively weaker relationship between $S$. aureus and IL-10 levels suggests other bacterial species may also induce IL-10 production as a common survival strategy in CRS.

Key words: chronic rhinosinusitis, pathophysiology of CRS, bacterial immune evasion, IL-10, Staphylococcus aureus

\section{Introduction}

Chronic rhinosinusitis (CRS) is an epidemiologically important chronic inflammatory disease process with significant, welldocumented economic implications ${ }^{(1,2)}$. Moreover, CRS confers a significant negative impact on patient quality of life analogous to that of congestive heart failure and chronic obstructive pulmonary disease ${ }^{(3)}$. Yet despite the commonality and severity of CRS, little is known about the exact pathophysiologic mecha- nism underlying this disease process.

Amongst the multitude of stated pathophysiologic explanations for CRS, bacteria have always been shown to play a critical role, albeit via novel mechanisms. Recent CRS literature is increasingly highlighting immune evasion by bacteria as another factor contributing to development of disease. The earliest suggestion of bacteria employing immune evasion strategies to facilitate their persistence dates back less than 10 years with the first 
reported publication demonstrating the presence of bacterial biofilms in CRS ${ }^{(4)}$. Although the discovery of bacterial biofilms is centuries old, the notion that bacteria are evading immune detection by forming organized communities shrouded in a protective polysaccharide matrix has strongly resonated within the scientific community directing considerable research into diagnostic strategies and therapeutic eradication. Additional research suggests that bacterial immune evasion extends below the nasal mucosal surface with evidence of intramucosal bacterial microcolonies (S. aureus in particular) in CRS patients in the absence of a local immune response ${ }^{(5-7)}$.

S. aureus is a frequently implicated pathogen in CRS ${ }^{(8)}$. As a pathobiont (bacteria with the potential to behave as a pathogen or as a symbiotic organism), S. aureus is capable of colonizing hosts asymptomatically but can also cause severe infections under selected circumstances. The mechanisms underlying pathobiosis are unknown although certain theories have been suggested. The Madrenas group has shown that S. aureus may promote commensalism by triggering a TLR-2 dependent, PI3K/ AKT mediated IL-10 response that downregulates pro-inflammatory T-cell host responses ${ }^{\left({ }^{9-12)}\right.}$. Taken together, the above findings suggest that $S$. aureus may be locally modulating host immunity to elude destruction by inducing an IL-10 mediated host response.

In view of supporting this hypothesis, we sought to clarify whether elevated IL-10 levels is a feature of CRS. Our primary aim was to evaluate whether IL-10 gene expression levels differ in CRS compared to controls, and to verify this with immunohistochemistry. Our secondary aim was to probe potential mechanisms by verifying whether IL-10 levels correlate with S. aureus and CD8+ T cell levels.

\section{Materials and methods}

\section{Ethical approval}

This study was approved by the ethical review board of the University of Montreal Hospital Research Centre (CRCHUM). All subjects were aged 18 years or more and voluntarily participated following informed consent.

\section{Study subjects}

Patients meeting criteria for CRS as per published American Academy of Otolaryngology-Head and Neck Surgery guidelines ${ }^{(13)}$ and had previously failed at least one course of maximal medical therapy were recruited. Non-CRS controls were undergoing a transnasal endoscopic approach for access to structures of the orbit, lacrimal system or skull base. Exclusion criteria included history of immune deficiency or cystic fibrosis. No patient received oral corticosteroids or antibiotics (oral or topical) in the 30 days prior to surgery. Administration of intranasal corticoste- roids was ceased at least 14 days prior to surgery.

\section{Microarray analysis}

An epithelial sample was harvested intraoperatively from the anterior ethmoid bulla using a disposable gastrointestinal cytology brush (ConMed, Utica, NY, USA). RNA was then extracted from epithelial samples in order to create a complementary DNA (cDNA) library for microarray analysis using the RNeasy Mini Kit (Qiagen, MD, USA). Microarray analysis was performed using an Illumina HumanHT-12 Beadchip v3 (Illumina, San Diego, CA, USA) at our institution's genomics facility (Genome Quebec at McGill University, Montreal, QC, Canada) to assess for IL-10 gene expression. No confirmation with PCR could be performed because of a technical problem in laboratory where samples were unfortunately destroyed in processing.

\section{Biopsy preparation for Immunohistochemistry}

At the same time as brushing, simultaneous surgical biopsy samples were taken at the level of the anterior ethmoid bulla. Biopsy samples were immediately transported on dry ice in a moist sterile compress in a sterile container to the pathology lab situated within the same hospital. Samples were then cut into $5 \mathrm{~mm}^{2}$ sections, submerged in optimal cutting temperature compound $(\mathrm{OCT})$ and stored at $-80^{\circ} \mathrm{C}$. Frozen biopsies were cut into 5 micron sections then mounted and fixed on glass slides with ethanol/methanol $60 / 40 \%$. Slides were stored at $-80^{\circ} \mathrm{C}$ until use.

\section{Immunohistochemistry (IHC) staining}

Immunohistochemistry (IHC) staining was performed on frozen sections obtained from the CRS surgical biopsies and control groups. A modified immunoperoxidase method of immunohistochemistry was performed. Five micron frozen sections were thawed and rinsed in PBS followed with $0.2 \%$ Triton X100 in PBS for 15 minutes. Endogenous peroxidase activity was blocked with $3 \%$ hydrogen peroxide for 15 minutes at room temperature. The slides were washed in PBS and pretreated with universal blocking solution (Dako, Toronto, ON, Canada) for 30 minutes. Section slides were incubated overnight at $4^{\circ} \mathrm{C}$ with primary diluted goat anti-human IL-10 antibody (R and D Systems, Minneapolis, MN, USA), CD8 (Dako, Toronto, ON, Canada) and S. aureus (rabbit anti-human, ABCAM, Toronto, ON, Canada). The slides were rinsed and incubated with the appropriate biotinylated secondary antibody for 30 minutes at room temperature. After washing in PBS, Streptavidin/Horse Radish Peroxidase complex (Vector, Canada) was applied for 30 minutes at room temperature. The reaction result was visualized with DAB/hydrogen peroxide (DAB Kit, Dako, Toronto, ON, Canada). Sections were rinsed in distilled water, lightly stained with hematoxylin, dehydrated, cleared, and cover slipped. Samples processed with the same isotypes, but without primary antibody served as negative controls. 


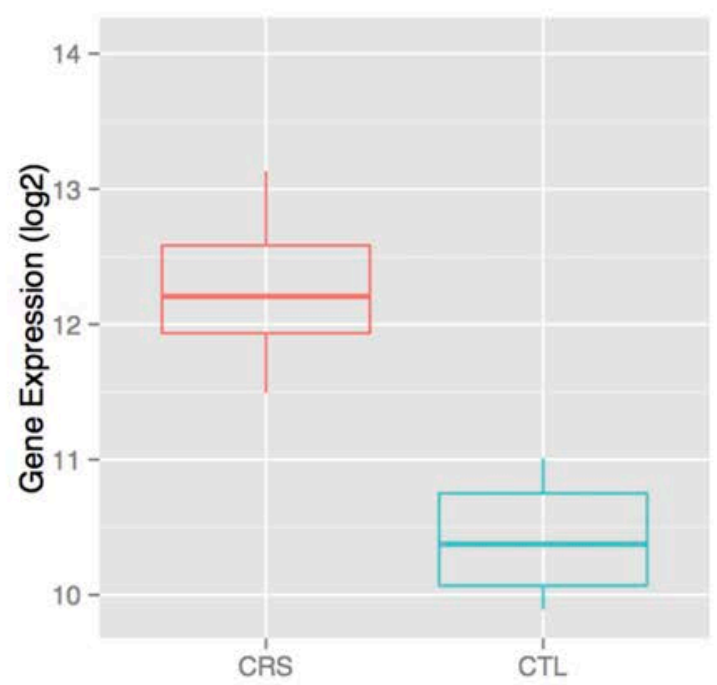

Figure 1. IL10 gene expression in CRS and control subjects. A box plot demonstrates the difference in IL-10 gene expression between CRS and CTL subjects (Fold Change $=3,64$ and FDR adjusted $p$ value $=1.27 \mathrm{x}$ 10-7). The $y$ axis represents the $\log 2$ gene expression.

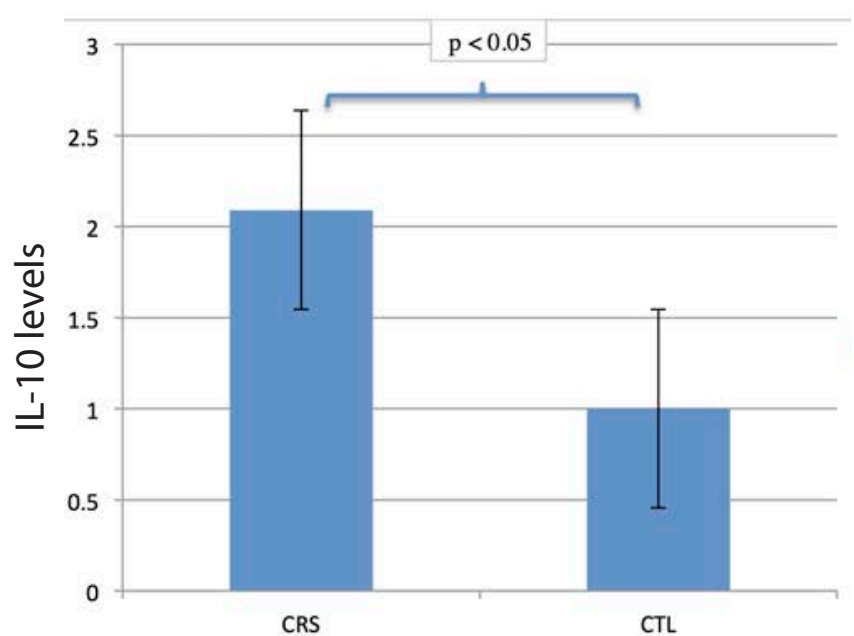

Figure 2. Comparison of IL-10 levels in CRS and controls. IL-10 is higher in CRS patients than in undiseased controls.

\section{Immunohistochemistry scoring}

IHC staining was graded by hand under an Olympus CX31 at $\times 400$ magnification for IL-10 and S. aureus intensity and at 200x magnification for $\mathrm{CD}^{+}$cell count. Only epithelial biopsies with sufficient areas of undamaged attached epithelium were subsequently analyzed for immunohistochemistry staining. Two different, blinded readers were assigned the task of scoring positive areas to avoid possible evaluator bias. For IL-10 and S. aureus, the intensity of coloration of the area of the epithelium and basement membrane was evaluated on a 5-point scale in 5 randomly selected mucosal fields with intact respiratory epithelium and the mean score calculated. The reference scale for grading intensity was developed by visualizing all samples in an unsupervised fashion, and identifying slides with minimal and maximal staining intensities. These were graded as 1 and 5, respectively.

For $\mathrm{CD}^{+}$, the number of positively staining cells were counted for five randomly selected high-powered fields with intact respiratory epithelium and the average determined. Any specimens in which difference in intensity varied by greater than a score of 0.5 or the cell count varied by more than $10 \%$ were discussed by the 2 observers to establish a consensus.

\section{Statistical analysis}

Differences in IL-10 gene expression was compared between CRS patients with active disease at the time of endoscopic sinus surgery (ESS) vs. controls. For gene expression, data analysis was performed using the Limma package from Bioconductor software. Results are expressed as a logarithmic fold change (FC) value with significance corresponding to a false discovery rate (FDR). Corrected FDR values of $\mathrm{p} \leq 0.05$ were deemed statistically significant.

For immunohistochemistry, the Wilcoxon rank test was used to assess the difference in the expression of IL-10 marker, CD8 ${ }^{+}$ T-cells and S. aureus in CRS and controls. A p value of $\leq 0.05$ was considered statistically significant. Pearson's correlation coefficient was employed to evaluate the correlation between levels of IL-10 and counts of CD8 ${ }^{+}$T-cells and ${ }^{\text {s. aureus. }}$. Correlation values were classified as no relationship ( $r=0$ to $<0.3$ ), moderate relationship ( $r=>0.3$ to 0.5 ) or strong relationship ( $r=>0.5$ to 1.0 ).

\section{Results}

\section{Microarray analysis}

A total of 22 epithelial brush samples were available for microarray analysis: 12 CRS [( 6 patients with CRS with nasal polyps (CRSwNP), 6 patients with CRS without nasal polyps (CRSsNP)] and 10 controls. CRS patients with active disease had a markedly higher expression of IL-10 compared to controls (FC = 3.464, adjusted $\mathrm{p}$ value $=1.273 \times 10-7$; Figure 1 ) .

\section{Immunohistochemistry}

All 22 patients ( $12 \mathrm{CRS}$ and $10 \mathrm{CTL}$ ) underwent surgical biopsies at the time of surgery. While all 12 CRS subjects had epithelial biopsies with sufficient areas of undamaged attached epithelium for analysis, biopsies from 5 of the CTL subjects had sufficient areas of undamaged epithelium, thus only 17 subjects could be assessed. 


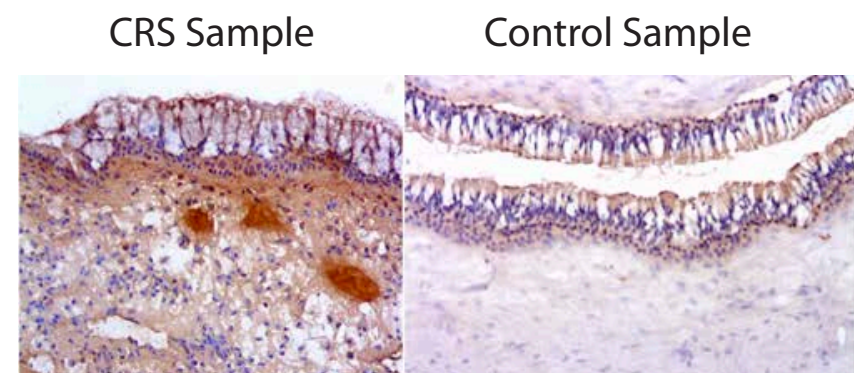

Figure 3. IL-10 IHC in sinus biopsies. (× 400). Representative samples illustrate differences between CRS and controls. In CRS samples, a high intensity of staining is seen in the subepithelial area with cluster formation, and to a lesser degree, within the epithelium. Staining intensity for $\mathrm{IL}-10$ is much less pronounced in controls.

Intraepithelial levels of IL-10 were significantly higher in CRS samples compared to controls ( $C T L=1.0$ and $C R S=2.09, \mathrm{p}<$ 0.05; Figure 2 and 3 ) as were levels of $S$. aureus ( $C T L=107.6$ and CRS $=267, p<0.05$; Figure 4 and 5). Intraepithelial CD8 ${ }^{+}$T-cell levels were significantly lower in CRS samples than in controls ( $C T L=164.6$ and $C R S=41.9, p<0.05$; Figure 4 and 6). Subgroup analysis of CRS patients with and without nasal polyposis revealed a reduction in $\mathrm{CD}^{+} \mathrm{T}$-cell levels in both groups (CRSsNP $=$ 30.3, CRSwNP = 58.8) with a corresponding increase in lgE levels (CRSsNP = 274.1, CRSwNP = 54.3). These findings appeared more pronounced in the CRSsNP group, though statistical comparison could not be performed due to the small sample size.

A strong inverse correlation was observed between intraepithelial CD8 ${ }^{+} T$ cell levels and intraepithelial IL-10 ( $r=-0.68$; Figure 7). Intraepithelial S. aureus strongly correlated with intraepithelial IL-10 levels ( $r=0.53$; Figure 8 ).

\section{Discussion}

Our findings suggest that elevated IL-10 levels are a feature of CRS. This may be implicated in a degree of immune suppression at the level of the sinus mucosa as increasing levels correlate with a downregulation of host $\mathrm{CD} 8^{+} \mathrm{T}$ cells. Both CRS patients with and without nasal polyposis demonstrated a reduction in $\mathrm{CD}^{+} \mathrm{T}$ cell activity with a corresponding increase in IgE levels suggesting that this immune evasion mechanism is present in both CRS disease subsets. This is a novel observation and suggests a new facet to elaboration of pathophysiologic mechanisms in CRS. While S. aureus is frequently implicated as the producer of virulence factors and toxins in CRS, in this instance it may not be the sole inducer of this response given the somewhat weaker correlation observed between S. aureus and IL-10 levels in CRS mucosa.

IL-10 is an important immunoregulatory cytokine with a pronounced inhibitory effect on myeloid cell function, thereby

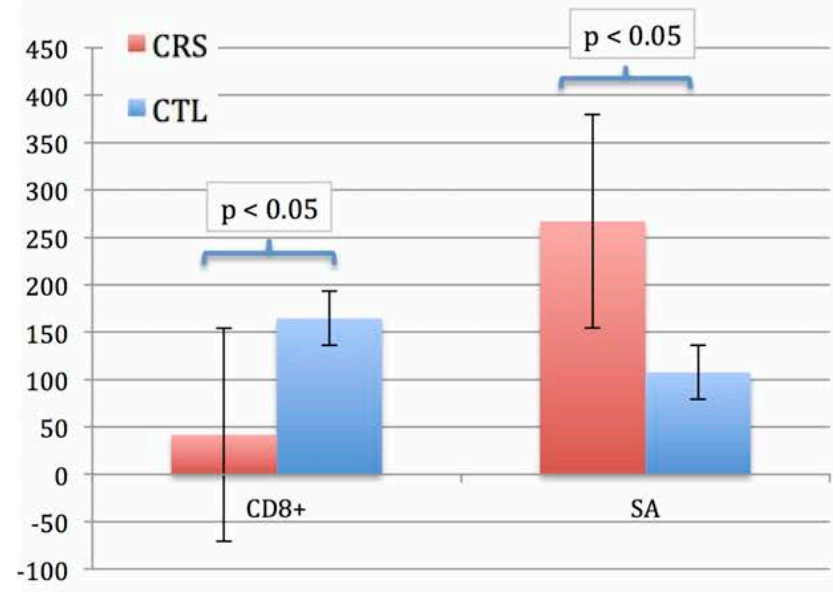

Figure 4. Comparison of CD8 ${ }^{+}$T-cells $\left(C D 8^{+}\right)$and S. aureus (SA) in CRS and controls. CRS epithelium is characterized by a depletion of $\mathrm{CD}^{+}$lymphocyte levels with a concomitant excess in S. aureus.

suppressing production of pro-inflammatory mediators and blunting T cell stimulation ${ }^{(14)}$. While we suspect that bacteria are implicated in inducing its production, it is not surprising that a weaker correlation is observed between IL-10 and S. aureus. While $S$. aureus is frequently implicated as a CRS pathogen, the bacterial makeup of CRS sinuses is typically polymicrobial. Therefore, the lack of a stronger relationship between elevated IL-10 and S. aureus suggests that local immunomodulation via IL-10 induction may be a common mechanism exploited by other bacterial pathogens contributing to the pathogenesis of CRS.

Previous work has hinted at a potential role for IL-10 related pathways in CRS. In a study by Plager et al. investigating differential gene expression in the sinonasal mucosa of asthmatic CRS patients with nasal polyposis compared to non-CRS patients, IL-10 signaling was one of the top canonical pathways identified ${ }^{(15)}$. Likewise, Kim et al. ${ }^{(16)}$ performed a genetic association study of IL-10 promoter polymorphisms by extracting genomic DNA from the peripheral blood of asthma patients with (AERD) or without aspirin hypersensitivity. A high IL-10 producing allele (IL-10-1082G) was identified as a risk factor for AERD. Moreover, when AERD subjects were stratified for CRS, a significant association was found with the IL-10 polymorphism (IL-10-1082G) and a polymorphism in TGF- $\beta 1$. The greatest distinction between this body of work and our own is that a genetic etiology implies an inherited, pre-existing deficiency in immune function. While the possibility of a pre-existing deficiency in immune function is supported by the recent identification by our group of a small percentage of severe CRS patients with low circulating levels of $\mathrm{CD}^{+}{ }^{\mathrm{T}}$-cells ${ }^{(17)}$, the low frequency of these cases fails to account for the more frequent occurrence of CRS in the general population, estimated at $16 \%{ }^{(1)}$. An alternative hypothesis, supported in 


\section{CRS Sample}

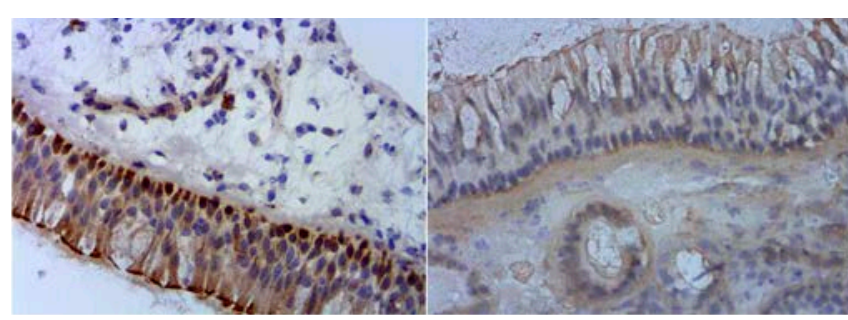

Figure 5. S. aureus IHC in sinus biopsies. $(\times 400)$. Representative samples illustrate differences between CRS and controls. In CRS samples, a higher intensity of staining is seen throughout the epithelial cells, with maximal presence at the basal pole.

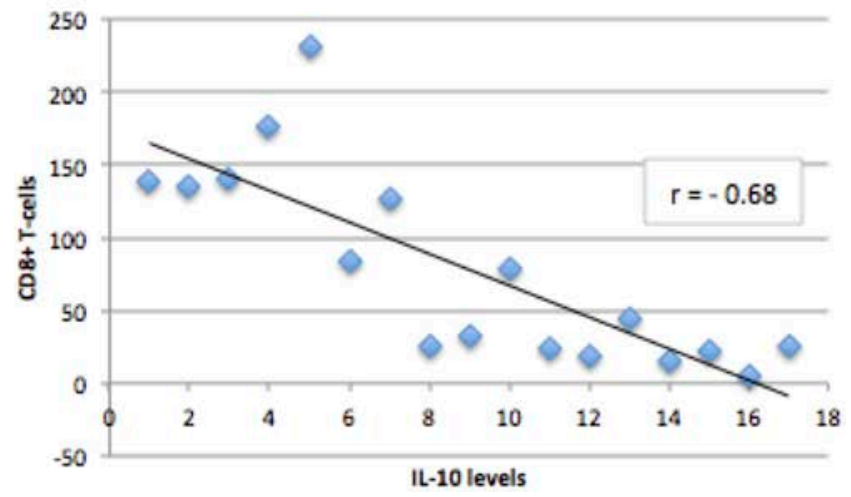

Figure 7. Correlation of IL-10 and CD8 ${ }^{+}$T-cell levels. A strong inverse correlation was observed between intraepithelial $C D 8^{+} \mathrm{T}$ cell levels and intraepithelial IL-10 $(r=-0.68)$.

part by the findings of our study, is that downregulation of the host immune response via induction of IL-10 is promoted locally by pathogenic bacteria as a common survival strategy. However, the presence of a weaker correlation between IL-10 and S. aureus in our study requires the identification of other CRS bacteria with IL-10 induction properties in order to fully substantiate this claim. Previous work by Chau et al. ${ }^{(9)}$ demonstrated that Coagulase-negative Staphylococcus (CNS), frequently isolated in the sinuses of CRS patients ${ }^{(18)}$, is also capable of inducing high levels of IL-10. Although laboratory strains of CNS were employed in their study, it is reasonable to assume that clinical CNS isolates may likewise induce high levels of IL-10. Further research must therefore be directed at immunoprofiling these and other frequently implicated bacterial organisms in CRS.

Although in the current context, IL-10 induction is associated with the manifestation of CRS, we do not believe that IL-10 in it of itself is detrimental to the sinus environment. Rather, an IL-10 induction capacity procures a survival advantage for resident bacteria irrespective of underlying pathogenicity. If pathogenic bacteria exploit this strategy, they may then express their viru-
CRS Sample

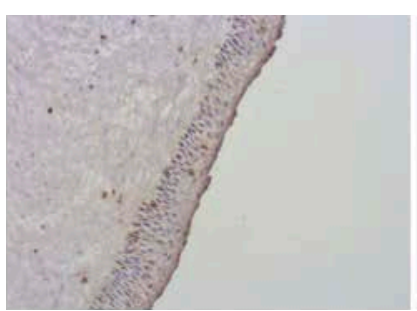

\section{Control Sample}

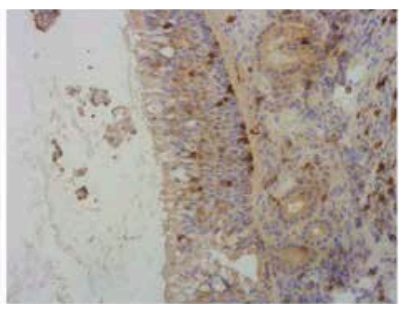

Figure 6. CD8 $8^{+}$lymphocyte IHC in sinus biopsies. (× 200). Representative samples illustrate differences between CRS and controls. A relative depletion of $\mathrm{CD}^{+} \mathrm{T}$-cells is seen in CRS compared to pronounced $\mathrm{CD} 8^{+}$ T-cell infiltration in healthy tissue.

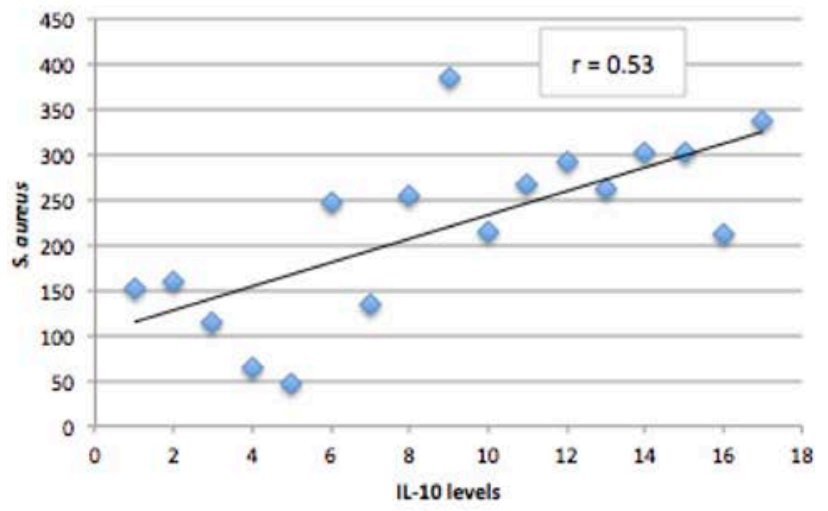

Figure 8. Correlation of IL-10 levels and S. aureus. Intraepithelial S. aureus strongly correlated with intraepithelial IL-10 levels ( $r=0.53)$.

lent factors that ultimately gives rise to the disease state. The implication of $S$. aureus virulence factors in CRS such as staphylococcal enterotoxins or "superantigens" has been extensively documented in CRS, supporting this concept ${ }^{(19)}$.

Findings from the rheumatologic and gastrointestinal literature have highlighted, much to the contrary, IL-10's role as an essential regulator of the immune response to the extent that deficiencies in this cytokine predispose to a pro-inflammatory disease state ${ }^{(20)}$. Polymorphisms in the IL-10 gene region resulting in deficient IL-10 production have been associated with systemic lupus erythematosis ${ }^{(21)}$, Behcet's disease ${ }^{(22,23)}$, inflammatory bowel disease (IBD) ${ }^{(24)}$, type 1 diabetes ${ }^{(25)}$ and severe juvenile rheumatoid arthritis ${ }^{(26)}$. Modulation of IL-10 for the management of IBD is currently being explored as a therapeutic alternative with several ongoing clinical trials investigating the administration of systemic IL-10 as well as genetically, high IL-10 inducing probiotics ${ }^{(27,28)}$.

The above research offers an additional layer of insight into our study's findings that will serve as groundwork for research by 
our group in the immediate future. While IL-10 may be manipulated by pathogenic bacteria involved in CRS, harvesting IL-10's anti-inflammatory properties for therapeutic means represents a novel management strategy for CRS. Reported success of probiotics in alleviating chronic inflammatory conditions such as IBD is attributed to probiotic induction of IL-10 ${ }^{(29)}$. We have recently identified 2 well tolerated, probiotic strains with IL-10 inducing capacity with the intention that these strains be topically administered in CRS patients. The goal would be to reconstitute the sinus microbiome of CRS patients with these well tolerated probiotic strains thereby competitively inhibiting the action of IL-10 inducing pathogenic bacteria.

\section{Conclusion}

Local bacterial immunomodulation (i.e. downregulation of $\mathrm{CD}^{+}$ T-cells) via IL-10 represents a novel pathophysiologic mechanism for CRS. While IL-10 levels correlate to a weaker degree with S. aureus levels, we suspect this is a common mechanism employed by other bacteria involved in CRS that merits further research.

\section{Authorship contribution}

JS: drafting, revision and final approval of manuscript, data acquisition, analysis and interpretation, oral presentation of research; SA: final approval of manuscript, data acquisition, analysis and interpretation; LME: final approval of manuscript, data acquisition, analysis and interpretation; SAR: final approval of manuscript, data acquisition, analysis and interpretation JM: final approval of manuscript, data acquisition, analysis and interpretation; MD: study design, drafting, revision and final approval of manuscript, data acquisition, analysis and interpretation.

\section{Conflict of interest}

None.

\section{References}

1. Anand VK. Epidemiology and economic impact of rhinosinusitis. Ann Otol Rhinol Laryngol Suppl. 2004;193:3-5.

2. Bhattacharyya N. Incremental health care utilization and expenditures for chronic rhinosinusitis in the United States. Ann Otol Rhinol Laryngol. 2011;120(7):423-7.

3. Macdonald KI, McNally JD, Massoud E. The health and resource utilization of Canadians with chronic rhinosinusitis. Laryngoscope. 2009;119(1):184-9.

4. Cryer J, Schipor I, Perloff JR, Palmer JN Evidence of bacterial biofilms in human chronic sinusitis. ORL J Otorhinolaryngol Relat Spec. 2004;66(3):155-8.

5. Clement $S$, Vaudaux $P$, Francois $P$, et al. Evidence of an intracellular reservoir in the nasal mucosa of patients with recurrent Staphylococcus aureus rhinosinusitis. J Infect Dis. 2005;192(6):1023-8.

6. Corriveau MN, Zhang N, Holtappels G, Van Roy N, Bachert C. Detection of Staphylococcus aureus in nasal tissue with peptide nucleic acid-fluorescence in situ hybridization. Am J Rhinol Allergy. 2009:23(5):461-5.

7. Wood AJ, Fraser JD, Swift S, PattersonEmanuelson EA, Amirapu S, Douglas RG. Intramucosal bacterial microcolonies exist in chronic rhinosinusitis without inducing a local immune response. Am J Rhinol Allergy. 2012;26(4):265-70.

8. Chin CW, Yeak CL, Wang DY. The microbiology and the efficacy of antibiotic-based medical treatment of chronic rhinosinusitis in Singapore. Rhinology. 2010;48(4):433-7.

9. Chau TA, McCully ML, Brintnell W, et al. Toll-like receptor 2 ligands on the staphylococcal cell wall downregulate superantigen-induced $T$ cell activation and prevent toxic shock syndrome. Nature Med.
2009;15(6):641-8.

10. Frodermann V, Chau TA, Sayedyahossein S, Toth JM, Heinrichs DE, Madrenas J. A modulatory interleukin-10 response to staphylococcal peptidoglycan prevents Th1/Th17 adaptive immunity to Staphylococcus aureus. J Infect Dis. 2011;204(2):253-62.

11. Mele T, Madrenas J. TLR2 signalling: At the crossroads of commensalism, invasive infections and toxic shock syndrome by Staphylococcus aureus. Int J Biochem Cell Biol. 2010;42(7):1066-71

12. Peres AG, Madrenas J. The broad land scape of immune interactions with Staphylococcus aureus: from commensalism to lethal infections. Burns. 2013;39(3):380-8.

13. Benninger MS, Ferguson BJ, Hadley JA, et al. Adult chronic rhinosinusitis: definitions, diagnosis, epidemiology, and pathophysiology. Otolaryngol Head Neck Surg. 2003;129(3 Suppl):S1-32.

14. Beebe AM, Cua DJ, de Waal Malefyt R. The role of interleukin-10 in autoimmune disease: systemic lupus erythematosus (SLE) and multiple sclerosis (MS). Cytokine Growth Factor Rev. 2002;13(4-5):403-12.

15. Plager DA, Kahl JC, Asmann YW, et al. Gene transcription changes in asthmatic chronic rhinosinusitis with nasal polyps and comparison to those in atopic dermatitis. Plos one. 2010;5(7):e11450.

16. Kim SH, Yang EM, Lee HN, Cho BY, Ye YM, Park HS. Combined effect of IL-10 and TGFbeta1 promoter polymorphisms as a risk factor for aspirin-intolerant asthma and rhinosinusitis. Allergy. 2009;64(8):1221-5.

17. Gabra N, Alromaih S, Endam LM, et al. Clinical features of cytotoxic CD8+ T-lymphocyte deficiency in chronic rhinosinusitis patients: a demographic and functional study.Int Forum Allergy Rhinol.
2014;4(6):495-501

18. Schlosser RJ, London SD, Gwaltney JM, Jr. Gross CW. Microbiology of chronic frontal sinusitis. Laryngoscope. 2001;111(8):1330-2.

19. Bachert C, Zhang N, Patou J, van Zele T, Gevaert P. Role of staphylococcal superantigens in upper airway disease. Curr Opin Allergy Clin Immunol. 2008;8(1):34-8.

20. Kubo M, Motomura Y. Transcriptional regulation of the anti-inflammatory cytokine IL-10 in acquired immune cells. Front Immunol. 2012;3:275.

21. Gateva V, Sandling JK, Hom G, et al. A largescale replication study identifies TNIP1, PRDM1, JAZF1, UHRF1BP1 and IL10 as risk loci for systemic lupus erythematosus. Nature Gen. 2009:41(11):1228-33.

22. Remmers EF, Cosan F, Kirino $Y$, et al. Genome-wide association study identifies variants in the MHC class I, IL10, and IL23RIL12RB2 regions associated with Behcet's disease. Nature Gen. 2010;42(8):698-702.

23. Mizuki N, Meguro A, Ota M, et al. Genomewide association studies identify IL23RIL12RB2 and IL10 as Behcet's disease susceptibility loci. Nature Gen. 2010;42(8):7036.

24. Franke $A$, Balschun $T$, Karlsen $\mathrm{TH}$, et al. Sequence variants in IL10, ARPC2 and multiple other loci contribute to ulcerative colitis susceptibility. Nature Gen. 2008;40(11):1319-23.

25. Barrett JC, Clayton DG, Concannon $P$, et al. Genome-wide association study and meta-analysis find that over 40 loci affect risk of type 1 diabetes. Nature Gen. 2009;41(6):703-7.

26. Crawley E, Kay R, Sillibourne J, Patel $P_{\text {, }}$ Hutchinson I, Woo P. Polymorphic haplotypes of the interleukin-10 5' flanking region determine variable interleukin-10 transcription and are associated with par- 
ticular phenotypes of juvenile rheumatoid arthritis. Arthritis Rheum. 1999;42(6):1101-8.

27. Braat $H$, Peppelenbosch H, W. HD Interleukin-10-based therapy for inflammatory bowel disease. Expert Opin Biol Ther. 2003;3(5):725-31.

28. Mukerji SS, Pynnonen MA, Kim HM, Singer A, Tabor M, Terrell JE. Probiotics as adjunctive treatment for chronic rhinosinusitis: a randomized controlled trial. Otolaryngol Head Neck Surg. 2009;140(2):202-8.

29. Pessi T, Sutas Y, Hurme M, Isolauri E. Interleukin-10 generation in atopic children following oral Lactobacillus rhamnosus GG. Clin Exp Allergy. 2000;30(12):1804-8.
Dr. Martin Desrosiers

Department of Otolaryngology

Head \& Neck Surgery

Université de Montréal

3840 Rue Saint-Urbain

Pavillon Marie-Morin, local 4-423

Montréal, Québec
Canada H2W 1 T8
Tel: (514)890-8000 ext 14170

Fax: (514)412-7246

E-mail: desrosiers_martin@hotmail.com 